\title{
Trend analysis of hydrological parameters of Ganga River
}

\author{
Mohammad Zakwan $^{1}$ (D) Zulfequar Ahmad $^{1}$
}

Received: 19 August 2020 / Accepted: 2 January 2021 / Published online: 25 January 2021

(C) Saudi Society for Geosciences 2021

\begin{abstract}
Ganga River basin, being the largest river basin in India, adheres with its social and spiritual importance for the country. Although many studies have been conducted on the Ganga basin, however, trend observed in flow and sediment yield in Ganga River has been scarcely studied. Alteration in flow pattern and sediment transport in rivers brings remarkable impact on river geomorphology and entire ecosystem of the region. The present paper attempts to identify trends observed in Ganga River in terms of annual maximum and annual minimum discharges, water and sediment yield during monsoon season. The trend analysis was accomplished by performing the Mann-Kendall (M-K), Sen's slope, and innovative trend analysis at various gauging sites along the river for the monsoon months. Significance of trend tests were tested at $5 \%$ significance level. Innovative trend analysis (ITA) revealed non-monotonicity within the time series and provided more detailed understanding of changes in hydrological changes. While observed annual maximum discharge showed a negative trend at almost all the sites, annual minimum discharge showed positive trend at gauging sites upstream of confluence of Yamuna River. Data of discharge and sediment load for monsoon months also revealed declining trends at most of the gauging sites. Water and sediment yields at all sites except Gandhighat showed a negative trend. Decline in trend of sediment and water yield is more pronounced in Western Ganga Plain (WGP) as compared to Eastern Ganga Plain (EGP). Incorporating these trends can be helpful for various water management projects in the future. Both climatic factors and human intervention appear to be responsible for the alteration in flow pattern of Ganga River.
\end{abstract}

Keywords Trend analysis · Ganga River · Mann-Kendall test · Sen's slope · Innovative trend analysis

\section{Introduction}

River systems are important geological agent. The transport of riverine sediment load from the continental land mass to the oceans is an important component of global biogeochemical cycle (Millimen and Meade 1983; Khattab and Merkel 2014). Alteration in climatic conditions directly affect the hydrologic cycle which may be observed in the form of variability of rainfall intensity, timing, or depth. Scientists all over the world are trying to understand the behavior of rivers through the years in view of the increasing anthropogenic and climatic influence (Khazaei et al. 2019). A number of studies have been

Responsible Editor: Broder J. Merkel

Mohammad Zakwan

zakwancivil@gmail.com

Zulfequar Ahmad

zulfifce@gmail.com

1 Civil Engineering Department, IIT Roorkee, Roorkee, India conducted to assess the trends in streamflow and sediment yield all over the world (Elouissi et al. 2017; Benzater et al. 2019).

Walling and Fang (2003) conducted trend analysis on 145 rivers around the world of which around $50 \%$ of the sediment load records showed statistically significant upward or downward trend. Similarly, Milliman et al. (2008) analyzed the water yield of 137 rivers and reported that more than one third of river presented alteration of more than $30 \%$ in water yield. However, total water draining to the global ocean remained constant as the decrease in cumulative discharge from midlatitude rivers was balanced by the increase in discharge of high-latitude rivers. Lu et al. (2003) analyzed the seasonal time series of discharge and sediment load in several tributaries of Yangtze River, China, the longest river in Asia, and reported remarkable alteration in the hydrological parameters, thereby projecting reasonable concern about flooding and water scarcity in different regions of Yangtze River basin. Rivers in central Japan experienced a significant decline in sediment load over the last few decades; however, during the same period, streamflow did not exhibit any remarkable trend (Siakeu et al. 2004). 
Apart from the abovementioned studies, hydrological behavior of Indian rivers has also been vastly studied (Arora et al. 2014; Subramanian 1996). Abbas and Subramanian (1984) determined the sediment load in River Ganga at Farakka Barrage and found it to be eight times the world average erosion rate as stated by Millimen and Meade (1983). Various levels of aggradation and degradation study have also been conducted in Indian rivers (Roy and Sinha 2007, 2014; Muzzammil et al. 2018; Pandey et al. 2018; Zakwan 2018). Tandon et al. (2006) concluded that in Western Ganga Plains, fluvial sedimentation is strongly affected by variation in monsoonal rainfall regime. In a study regarding sediment flux over IndoGangetic plains, Northern Bihar, Sinha and Friend (1994) observed that suspended sediment plot over a 10 -year period showed variation from year to year as well as a shift in total sediment discharge from downstream to upstream since 1986. Chakrapani and Saini (2009) studied the temporal variation in sediment in Alaknanda and Bhagirathi Rivers (forming Ganga) and reported large variation in suspended sediment load in monsoon and non-monsoon season.

Although ample work has been performed in terms of geomorphological study of Ganga River, quantitative detection of trend in flow and sediment data of the river has seldom been analyzed. This may be attributed to the absence of large database pertaining to sediment and flow data of the river. This paper aims to apply non-parametric statistical test, namely, the Mann-Kendall, Sen's slope, and innovative trend analysis (ITA), on various gauging sites along the Ganga River to detect presence of significant trend in annual maximum discharge, annual minimum discharge, monthly discharge, water yield, and suspended sediment yield for monsoon months of June to October.

\section{Ganga River basin}

With a total length of $2525 \mathrm{~km}$ and catchment area of 8,61,542 $\mathrm{km}^{2}$, Ganga River is one of the longest rivers in India. Ganga River basin spreads between $73^{\circ} 2^{\prime}$ to $89^{\circ} 5^{\prime} \mathrm{E}$ and $21^{\circ} 6^{\prime}$ to $31^{\circ} 21^{\prime} \mathrm{N}$. Rivers Alaknanda and Bhagirathi join at Devprayag to form Ganga which then flows into the Bay of Bengal. The principal tributaries joining the river are Yamuna, Sone, Ghaghara, Gandak, and Kosi. Ganga River caters towards water supply, hydroelectric, irrigation, and drinking water needs. It has a varying climate ranging from tropical, sub-tropical, temperate, to alpine and possesses a mean annual temperature of $24{ }^{\circ} \mathrm{C}$. The basin receives nearly $80 \%$ of its total rainfall in the monsoon period of June-October and its average annual rainfall varies from 400 to $2000 \mathrm{~mm}$ (Zakwan et al. 2018).

\section{Data set and methodology}

\section{Data set}

Hydrologic data at various gauging sites along Ganga was procured from Central Water Commission (CWC). Figure 1 shows the location and chainage of these gauging sites. Apart from that, mean discharge and suspended sediment load of the river are also observed at these gauging sites. The mean values are reported as an average over 10 days of the month for the monsoon season June-October. The data used in this study along with the time period of data availability is tabulated in Table 1.

\section{Methodology of trend analysis}

The non-parametric Mann-Kendall (Mann 1945; Kendall 1975) and Sen's slope (Sen 1968) were applied in this study to determine presence of trend in flow and suspended sediment load time series. These non-parametric tests do not require data series to follow normal distribution and yet their results are comparable with other parametric trend tests (Yue et al. 2002; Ebadati et al. 2014; Tirkey et al. 2020). MATLAB program was used for obtaining trends through the MannKendall and Sen's slope, while Excel spreadsheet was used for obtaining trends of ITA.

\section{The Mann-Kendall test}

The Mann-Kendall test is a statistical test widely used for the analysis of trend in climatologic and in hydrologic time series. According to this test, the null hypothesis $H_{0}$ assumes that there is no trend (the data is independent and randomly ordered) and this is tested against the alternative hypothesis $H_{1}$, which assumes that there is a trend. $X_{i}$ and $X_{j}$ are two subsets of data where $i=1,2,3, \ldots, n-1$ and $j=i+1, i+2, i+3, \ldots$, $n$.

The Mann-Kendall $S$ statistic is computed as follows:

$S=\sum_{i=1}^{n-1} \sum_{j=i+1}^{n} \operatorname{sign}\left(X_{j}-X_{i}\right)$

$\operatorname{Sign}\left(X_{j}-X_{i}\right)=\left\{\begin{array}{c}1 \text { if } X_{j}-X_{i}>0 \\ 0 \text { if } X_{j}-X_{i}=0 \\ -1 \text { if } X_{j}-X_{i}<0\end{array}\right.$

The variance $\left(\sigma^{2}\right)$ for the $S$ statistic is defined by:

$\sigma^{2}=\frac{n(n-1)(2 n+5)-\sum_{i=1}^{m} t_{i}\left(t_{i}-1\right)(2 t+5)}{18}$ 
Table 1 Data availability period for different gauging sites

\begin{tabular}{|c|c|c|c|c|c|}
\hline \multirow[t]{2}{*}{ Chainage } & \multirow[t]{2}{*}{ Gauging site } & \multicolumn{3}{|l|}{ Discharge } & \multirow{2}{*}{$\begin{array}{l}\text { Suspended sediment load (10 } \\
\text { daily average) }\end{array}$} \\
\hline & & $\begin{array}{l}\text { Annual } \\
\text { maximum }\end{array}$ & $\begin{array}{l}\text { Annual } \\
\text { minimum }\end{array}$ & $\begin{array}{l}\text { Average (10 } \\
\text { daily) }\end{array}$ & \\
\hline 0 & Garhmukteshwar & 1967-2014 & $1967-2014$ & $1967-2014$ & 1974-2004 \\
\hline 147 & Kachlabridge & 1971-2014 & $1971-2014$ & $1972-2014$ & $1973-2015$ \\
\hline 263 & Fatehgarh & 1972-2014 & $1972-2014$ & $1972-2014$ & $1978-2015$ \\
\hline 348 & Ankinghat & 1968-2014 & $1968-2014$ & 1968-2014 & $1977-2014$ \\
\hline 416 & Kanpur & 1960-2014 & 1960-2014 & 1960-2014 & $1975-2014$ \\
\hline 489 & Bhitaura & 1970-2014 & 1970-2014 & $1971-2014$ & $1978-2014$ \\
\hline 580 & Shahzadpur & 1960-2014 & 1960-2014 & 1960-2014 & 1963-2014 \\
\hline 652 & Allahabad & 1970-2014 & 1970-2014 & 1970-2014 & 1973-2014 \\
\hline 774 & Mirzapur & 1976-2014 & $1976-2014$ & 1979-2014 & 1980-2014 \\
\hline 858 & Varanasi & 1960-2014 & 1960-2014 & 1960-2014 & $1962-2014$ \\
\hline 1017 & Buxar & 1960-2014 & $1960-2014$ & 1960-2014 & $1996-2015$ \\
\hline 1165 & Gandhighat & 1965-2014 & $1965-2014$ & 1965-2014 & $1997-2013$ \\
\hline 1262 & Hathidah & 1961-2014 & $1961-2014$ & 1961-2014 & 1996-2013 \\
\hline 1432 & Azamabad & 1960-2014 & 1960-2014 & 1960-2014 & $1996-2015$ \\
\hline 1572 & Farakka & 1960-2014 & $1960-2014$ & 1960-2014 & 1995-2014 \\
\hline
\end{tabular}

The standard test statistic $Z_{s}$ is calculated as follows:

$Z_{S}=\left\{\begin{array}{l}\frac{S-1}{\sigma} \text { for } S>0 \\ 0 \text { for } S=0 \\ \frac{S+1}{\sigma} \text { for } S<0\end{array}\right.$

where $m$ is the number of unique values (without duplicates) and $t_{i}$ is the frequency of the $i$ th value. If $\left|Z_{s}\right|$ is greater than $Z_{\alpha / 2}$, where $\alpha$ represents the chosen significance level ( $5 \%$ with $\left.Z_{0.025}=1.96\right)$, then the null hypothesis is invalid implying that the trend is significant.

\section{Sen's slope estimator}

Sen (1968) proposed the non-parametric Sen's slope statistics. Slope for each pair may be calculated as follows:

$Q_{i}=\frac{\left(X_{j}-X_{k}\right)}{j-k}$ where $(j>k)($ for $i=1,2,3, \ldots, n)$

where $X_{j}$ and $X_{k}$ are the data values at times $j$ and $k(j>k)$, respectively.

Sen's slope estimator can then be calculated as follows:

$Q_{\text {med }}=\left\{\begin{array}{c}Q[(n+1) / 2] \text { if } n \text { is odd } \\ \frac{Q_{n / 2}+Q_{(n+2) / 2}}{2} \text { if } n \text { is even }\end{array}\right.$

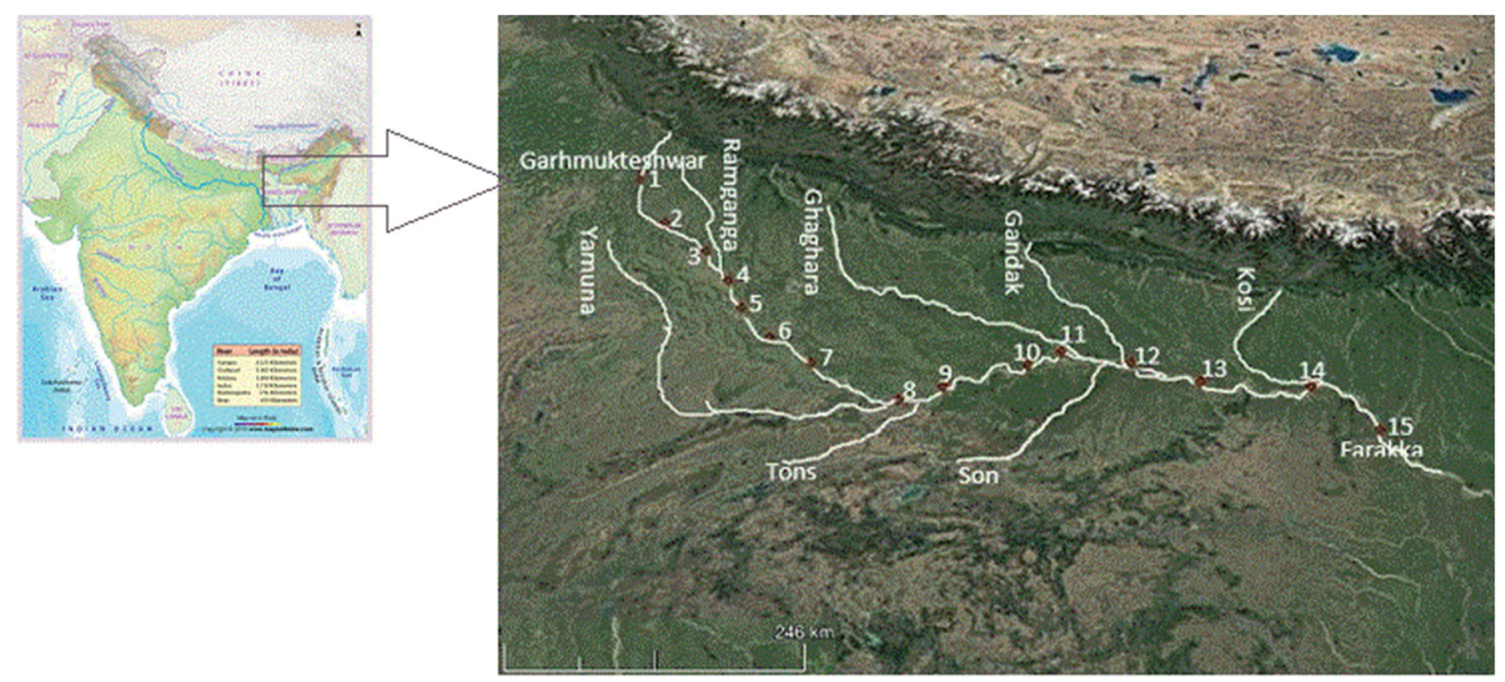

Fig. 1 Various gauging sites along the Ganga River 
The $Q_{\text {med }}$ sign reflects data trend, while its value indicates the steepness of the trend.

\section{Innovative trend analysis}

Şen (2012) presented an innovative trend analysis. The procedure for ITA may be summarized as follows:

Divide the entire time series into two equal halves. Calculate the average of both halves as $\overline{Y_{1}}$ and $\overline{Y_{2}}$. Arrange both halves of the time series in ascending order. Prepare a plot with first half of time series on abscissa and second half series on ordinate. Also plot the 1:1 $\left(45^{\circ}\right)$ line on the same plot. Relative position of scatter point with respect to $45^{\circ}$ line demarcates the trend. If all the points lie above the $45^{\circ}$ line, it will represent monotonically increasing trend; on the other hand, if all the points lie below the $45^{\circ}$ line, it will represent monotonically decreasing trend; otherwise, trend may not be monotonic (Elouissi et al. 2016).

The magnitude of trend may be calculated as follows

$s=\frac{2\left(\overline{Y_{2}}-\overline{Y_{1}}\right)}{n}$

Confidence limit (CL) of trend may be calculated using the following relationship (Șen 2017):

$C L_{(1-a)}=0+S_{c r i} \sigma_{s}$

where $s_{\text {cri }}$ is the critical slope and $\sigma_{\mathrm{s}}$ is the standard deviation slope.

$\sigma_{s}^{2}=\frac{8 \sigma^{2}\left(1-\rho_{Y_{2} Y_{1}}\right)}{n^{3}}$

where $\rho_{Y_{2}} Y_{1}$ is the cross-correlation coefficient of averages of two halves given by the following:

$\rho_{Y_{2} Y_{1}}=\frac{E\left(Y_{2} Y_{1}\right)-E\left(Y_{2}\right) E\left(Y_{1}\right)}{\sigma_{Y_{2}} \sigma_{Y_{1}}}$

Figure 2 explains the trend identification process of ITA. Figure 2 shows 1:1 line with first and second half of the time series on either axis. The plot shows low-, moderate-, and high-magnitude event. From the plotted points, the magnitude event trends can be understood distinctly. In this way, innovative trend analysis has an advantage over non-parametric tests, that non-parametric tests only reveal monotonic trend; however, it may be possible that hydrological events of different magnitude may have different trends. As can be observed from Fig. 2, low-magnitude events are trendless, while

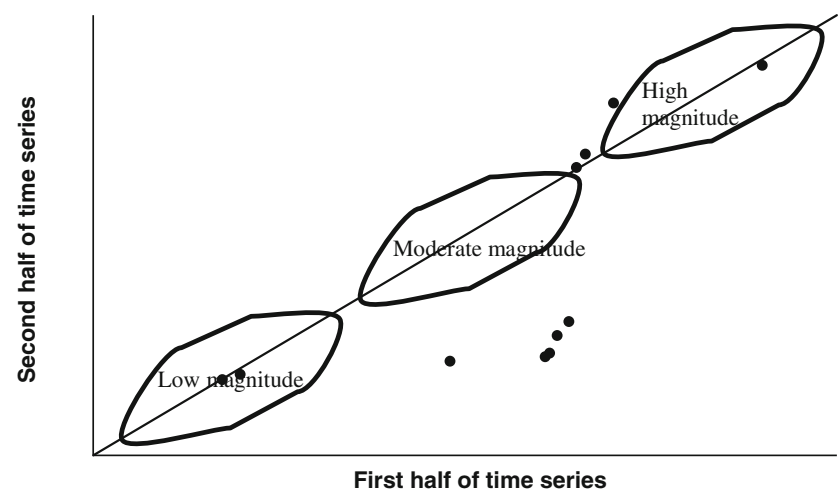

Fig. 2 Graphical representation of innovative trend analysis

moderate-magnitude events show significant negative trend and high-magnitude events show slight positive trend.

\section{Results and discussion}

To understand the general behavior of hydrological parameters of Ganga River, time series of annual maximum discharge, annual minimum discharge, monthly discharge, monthly sediment load, water and sediment yield for monsoon season at each gauging site were tested using non-parametric trend tests.

The test statistics obtained for the Mann-Kendall, Sen's slope test, and ITA for annual maximum and annual minimum discharge at various gauging sites are reported in Tables 2 and 3 respectively. A perusal of Table 2 reveals a significant negative trend in annual maximum discharge at Farakka, Buxar, Varanasi, Mirzapur, Allahabad, Shahzadpur, Kanpur, Fatehgarh, and Garhmukteshwar. It was observed that the slope of first half of the annual maximum discharge time series was much higher than the second half, reflecting decline in the annual maximum discharge. The highest rate of decline in annual maximum discharge was observed at Allahabad followed by Mirzapur and Shahzadpur. At Allahabad, the slope of first half of the time series was around $21,127 \mathrm{~m}^{3} / \mathrm{s} /$ year which declined to $14,052 \mathrm{~m}^{3} / \mathrm{s} /$ year. The trend slope obtained for ITA based on Eq. $7\left(-685 \mathrm{~m}^{3} / \mathrm{s} /\right.$ year $)$ was found to be greater than critical slope $\left(46.98 \mathrm{~m}^{3} / \mathrm{s} /\right.$ year $)$ reflecting significant trend at 5\% significance level. The Mann-Kendall test also revealed significant trend at Allahabad $(-4.73<-1.96)$. These high rates of fall in annual maximum discharge may be attributed to the drying up of River Yamuna as reported by Misra (2010). The decline in discharge in the upstream reach (Garhmukteshwar-Ankinghat) can be attributed to decline in discharge from Gangotri Glacier (Jain 2008).

A positive trend was observed in annual minimum discharge at upstream gauging sites of Garhmukteshwar, Kachlabridge, Fatehgarh, Ankinghat, Kanpur, Bhitaura, and Shahzadpur; however, a negative trend is observed 
Table 2 Result of trend tests at various sites for annual maximum discharge

\begin{tabular}{|c|c|c|c|c|c|c|c|}
\hline \multirow[t]{3}{*}{ Gauging sites } & \multicolumn{7}{|c|}{ Annual maximum discharge } \\
\hline & \multirow{2}{*}{$\begin{array}{l}\mathrm{M}-\mathrm{K} \\
Z_{s}\end{array}$} & \multicolumn{6}{|l|}{ ITA } \\
\hline & & Sen's slope & First half mean $\left(\overline{Y_{1}}\right)$ & Second half mean $\left(\overline{Y_{2}}\right)$ & Standard deviation $(\sigma)$ & Trend slope $(s)$ & Critical slope (Scr) \\
\hline Garhmukteshwar & -2.40 & -45.99 & 4885.11 & 3982.02 & 1882.19 & -39.26 & 8.70 \\
\hline Kachlabridge & -1.13 & -23.80 & 5823.42 & 5255.55 & 2035.86 & -27.09 & 8.42 \\
\hline Fatehgarh & -2.90 & -62.29 & 4619.31 & 4001.76 & 2149.99 & -29.41 & 10.85 \\
\hline Ankinghat & -1.76 & -63.48 & 7185.97 & 5568.13 & 2793.11 & -77.04 & 12.19 \\
\hline Kanpur & -2.95 & -74.08 & 7681.23 & 6726.19 & 3281.95 & -39.79 & 17.49 \\
\hline Bhitaura & -1.97 & -73.08 & 6896.84 & 6259.51 & 4201.39 & -30.33 & 22.37 \\
\hline Shahzadpur & -5.23 & -133.54 & 8616.78 & 4157.99 & 3675.75 & -185.78 & 11.99 \\
\hline Allahabad & -4.53 & -700.38 & $35,531.12$ & $21,127.23$ & $14,052.62$ & -685.85 & 46.98 \\
\hline Mirzapur & -3.63 & -601.54 & $33,788.85$ & $22,054.23$ & $13,564.81$ & -617.62 & 65.81 \\
\hline Varanasi & -2.45 & -227.28 & $31,319.52$ & $23,689.52$ & 9972.47 & -317.92 & 22.47 \\
\hline Buxar & -2.74 & -177.78 & $30,804.48$ & $24,932.23$ & 8868.33 & -244.67 & 37.47 \\
\hline Gandhighat & 0.38 & 73.42 & $45,353.36$ & $47,114.63$ & $15,111.32$ & 73.39 & 34.18 \\
\hline Hathidah & -1.72 & -194.29 & $52,683.02$ & $47,281.23$ & $11,202.40$ & -225.05 & 21.64 \\
\hline Azamabad & -0.33 & -37.39 & $55,362.36$ & $49,762.1$ & $14,177.79$ & -233.23 & 52.45 \\
\hline Farakka & -3.55 & -465.0 & $57,852.45$ & $46,904.25$ & $13,125.06$ & -457.23 & 73.37 \\
\hline
\end{tabular}

Italicized values represent significant trend at 5\% significance level

downstream towards Allahabad and Buxar. Varanasi showed significant positive trend and an insignificant positive trend was also observed further downstream at Gandhighat and
Hathidah. The highest rate of change in annual minimum observed discharge was found at Fatehgarh followed by Garhmukteshwar and Kachlabridge. Farakka also showed a

Table 3 Result of trend tests at various sites for annual minimum discharge

\begin{tabular}{|c|c|c|c|c|c|c|c|}
\hline \multirow[t]{3}{*}{ Gauging sites } & \multicolumn{7}{|c|}{ Annual minimum discharge } \\
\hline & \multirow{2}{*}{$\begin{array}{l}\text { M-K } \\
Z_{s}\end{array}$} & \multicolumn{6}{|l|}{ ITA } \\
\hline & & Sen's slope & First half mean $\left(\overline{Y_{1}}\right)$ & Second half mean $\left(\overline{Y_{2}}\right)$ & Standard deviation $(\sigma)$ & Trend slope $(s)$ & Critical slope (Scr) \\
\hline Garhmukteshwar & 4.28 & 1.33 & 46.34 & 81.52 & 36.86 & 1.53 & 0.19 \\
\hline Kachlabridge & 3.80 & 0.21 & 10.26 & 15.68 & 6.69 & 0.26 & 0.05 \\
\hline Fatehgarh & 2.85 & 0.22 & 11.63 & 20.32 & 9.30 & 0.41 & 0.03 \\
\hline Ankinghat & 0.79 & 0.15 & 46.24 & 56.35 & 23.30 & 0.48 & 0.09 \\
\hline Kanpur & 1.45 & 0.22 & 50.86 & 52.19 & 20.66 & 0.06 & 0.07 \\
\hline Bhitaura & 2.18 & 0.66 & 53.01 & 71.38 & 24.17 & 0.87 & 0.13 \\
\hline Shahzadpur & 1.57 & 0.33 & 50.26 & 56.65 & 28.16 & 0.27 & 0.07 \\
\hline Allahabad & -2.02 & -1.16 & 192.92 & 168.34 & 66.81 & -1.17 & 0.40 \\
\hline Mirzapur & 0.00 & -0.02 & 179.08 & 205.16 & 71.54 & 1.37 & 0.68 \\
\hline Varanasi & 2.38 & 1.11 & 173.23 & 196.55 & 62.06 & 0.97 & 0.32 \\
\hline Buxar & 0.79 & 0.89 & 323.44 & 332.35 & 125.26 & 0.37 & 0.43 \\
\hline Gandhighat & 1.57 & 1.95 & 1164.41 & 1405.74 & 484.82 & 10.05 & 0.95 \\
\hline Hathidah & 0.33 & 1.52 & 1537.81 & 1688.52 & 501.07 & 6.28 & 1.32 \\
\hline Azamabad & -0.22 & -0.93 & 1688.66 & 1641.07 & 560.35 & -1.98 & 1.42 \\
\hline Farakka & -2.45 & -1.17 & 223.44 & 182.35 & 105.26 & -0.27 & 0.39 \\
\hline
\end{tabular}

Italicized values represent significant trend at 5\% significance level 
significant negative trend in annual minimum discharge. Generally, in the Ganga River basin, minimum discharge is observed in the pre-monsoon months (March, April, and May). Zakwan and Ara (2019) reported positive trend in rainfall for the pre-monsoon period which could be the reason behind increase in annual minimum discharge.

Ten daily average discharges were available at fifteen gauging sites for the monsoon period. Using the ten daily data total volumes of water crossing gauging sites in the monsoon period were calculated and trend analysis was performed on the observed water yield at fifteen gauging sites. The results of the Mann-Kendall, Sen's slope, and innovative trend tests are shown in Tables 4, 5, and 6 respectively. Almost all the gauging sites showed a negative trend in terms of sediment yield crossing the gauging sites during the monsoon period with Ankinghat, Kanpur, Shahzadpur, Allahabad, Varanasi, and
Farakka showing significantly negative trend. The upstream gauging sites Garhmukteshwar, Kanpur, and Shahzadpur also showed significant negative trend which may be attributed to decline in discharge from Gangotri Glacier as reported by Jain (2008). Also the annual rainfall received by the region has declined resulting in decline in discharge (Bisht et al. 2018; Zakwan and Ara 2019).

The general trend observed at all the gauging sites except Gandhighat was negative, indicating a lowering of volume of water along the river with time. In line with this general behavior, a significant negative trend with a decrease in rate of $1.1 \%$ is observed at Farakka gauging sites. This might be due to the presence of Farakka Barrage in the downstream.

This indicates a significant decrease of volume of suspended sediment crossing the gauging sites. The rate of decrease of suspended sediment load at Farakka is observed
Table 4 Results of trend analysis of monthly data for Mann-Kendal test

\begin{tabular}{|c|c|c|c|c|c|c|}
\hline Gauging sites & Quantity & June & July & August & September & October \\
\hline \multirow[t]{2}{*}{ Garhmukteshwar } & Discharge & -1.17 & -2.88 & -3.01 & -1.10 & 0.70 \\
\hline & Sediment load & -2.75 & -2.03 & -1.71 & -0.46 & -0.32 \\
\hline \multirow[t]{2}{*}{ Kachlabridge } & Discharge & -0.61 & -0.90 & -0.42 & -1.05 & 0.48 \\
\hline & Sediment load & -0.86 & -0.13 & 1.45 & 1.34 & 2.47 \\
\hline \multirow[t]{2}{*}{ Fatehgarh } & Discharge & -1.09 & -2.01 & -2.13 & -0.86 & -0.54 \\
\hline & Sediment load & -1.27 & -2.34 & -3.34 & -0.95 & 0.90 \\
\hline \multirow[t]{2}{*}{ Ankinghat } & Discharge & -1.45 & -2.75 & -2.18 & -0.90 & -0.29 \\
\hline & Sediment load & -1.92 & -3.82 & -3.77 & -3.04 & -0.25 \\
\hline \multirow[t]{2}{*}{ Kanpur } & Discharge & -0.78 & -2.50 & -1.83 & -1.77 & -1.22 \\
\hline & Sediment load & -1.88 & -2.53 & -2.57 & -1.71 & -0.83 \\
\hline \multirow[t]{2}{*}{ Bithaura } & Discharge & 0.13 & -1.19 & -0.94 & -0.84 & 0.52 \\
\hline & Sediment load & -1.01 & -0.82 & -1.04 & -1.72 & 0.57 \\
\hline \multirow[t]{2}{*}{ Shahzadpur } & Discharge & -0.39 & -3.50 & -4.44 & -3.85 & -1.44 \\
\hline & Sediment load & 0.43 & -4.93 & -5.82 & -4.90 & -2.39 \\
\hline \multirow[t]{2}{*}{ Allahabad } & Discharge & -2.17 & -3.12 & -3.63 & -3.43 & -1.11 \\
\hline & Sediment load & -0.48 & -2.74 & -3.24 & -3.08 & -0.29 \\
\hline \multirow[t]{2}{*}{ Mirzapur } & Discharge & 0.57 & -0.54 & -2.33 & -2.61 & -0.65 \\
\hline & Sediment load & 1.36 & 0.11 & -0.94 & -1.73 & 0.74 \\
\hline \multirow[t]{2}{*}{ Varanasi } & Discharge & 0.67 & -1.79 & -2.53 & -2.60 & -1.35 \\
\hline & Sediment load & -1.04 & -1.65 & -2.77 & -2.14 & -0.43 \\
\hline \multirow[t]{2}{*}{ Buxar } & Discharge & 0.29 & -1.12 & -2.21 & -1.22 & 0.13 \\
\hline & Sediment load & 1.12 & 0.14 & -1.12 & -1.12 & 1.09 \\
\hline \multirow[t]{2}{*}{ Gandhighat } & Discharge & 0.89 & -0.47 & -0.15 & 0.03 & 0.42 \\
\hline & Sediment load & 0.61 & 0.00 & 0.08 & -0.76 & 1.44 \\
\hline \multirow[t]{2}{*}{ Hathidah } & Discharge & -0.12 & -0.72 & -1.18 & -0.58 & 0.42 \\
\hline & Sediment load & 0.61 & 0.15 & 0.00 & -0.68 & -0.43 \\
\hline \multirow[t]{2}{*}{ Azamabad } & Discharge & -0.48 & 0.61 & -0.32 & 0.20 & 0.52 \\
\hline & Sediment load & -0.91 & -1.75 & -1.05 & -2.17 & -1.82 \\
\hline \multirow[t]{2}{*}{ Farakka } & Discharge & -0.80 & -1.09 & -2.85 & -2.62 & -0.88 \\
\hline & Sediment load & -0.03 & -2.30 & -2.56 & -3.21 & -2.76 \\
\hline
\end{tabular}

Italicized values represent significant trend at 5\% significance level 
Table 5 Sen's slope of trend analysis of monthly data

\begin{tabular}{|c|c|c|c|c|c|c|}
\hline Gauging sites & Quantity & June & July & August & September & October \\
\hline \multirow[t]{2}{*}{ Garhmukteshwar } & Discharge & -4.05 & -16.66 & -26.58 & -6.20 & 1.30 \\
\hline & Sediment load & $-12,856.09$ & $-67,702.13$ & $-87,173.17$ & $-10,179.10$ & -695.70 \\
\hline \multirow[t]{2}{*}{ Kachlabridge } & Discharge & -1.18 & -12.14 & -2.14 & -12.87 & 1.02 \\
\hline & Sediment load & -532.92 & -2774.14 & $90,495.23$ & $47,132.16$ & 7422.81 \\
\hline \multirow[t]{2}{*}{ Fatehgarh } & Discharge & -1.73 & -17.95 & -20.88 & -9.53 & -1.88 \\
\hline & Sediment load & -415.17 & $-28,141.29$ & $-81,928.55$ & $-19,346.88$ & 766.29 \\
\hline \multirow[t]{2}{*}{ Ankinghat } & Discharge & -2.71 & -25.72 & -39.62 & -12.84 & -1.63 \\
\hline & Sediment load & -708.71 & $-58,108.04$ & $-152,618.67$ & $-86,820.97$ & -659.95 \\
\hline \multirow[t]{2}{*}{ Kanpur } & Discharge & -0.88 & -18.79 & -30.09 & -24.90 & -5.87 \\
\hline & Sediment load & -394.17 & $-49,084.19$ & $-141,820.90$ & $-57,278.76$ & -3097.57 \\
\hline \multirow[t]{2}{*}{ Bithaura } & Discharge & 0.16 & -13.23 & -20.50 & -15.16 & 3.69 \\
\hline & Sediment load & -65.86 & -5754.70 & $-28,640.50$ & $-55,073.90$ & 2696.54 \\
\hline \multirow[t]{2}{*}{ Shahzadpur } & Discharge & -0.32 & -22.52 & -70.26 & -54.49 & -7.87 \\
\hline & Sediment load & 36.50 & $-31,777.76$ & $-144,149.12$ & $-82,317.42$ & -8564.58 \\
\hline \multirow[t]{2}{*}{ Allahabad } & Discharge & -43.99 & -1037.37 & -3198.83 & -2624.22 & -193.14 \\
\hline & Sediment load & -511.52 & $-98,908.26$ & $-493,203.15$ & $-340,687.76$ & -4053.39 \\
\hline \multirow[t]{2}{*}{ Mirzapur } & Discharge & 18.55 & -211.20 & -2994.59 & -2376.66 & -131.29 \\
\hline & Sediment load & 1503.62 & 6734.15 & $-238,566.10$ & $-381,178.09$ & $16,728.03$ \\
\hline \multirow[t]{2}{*}{ Varanasi } & Discharge & 10.43 & -401.98 & -1204.16 & -1457.91 & -183.93 \\
\hline & Sediment load & -34.55 & $-30,454.65$ & $-381,030.94$ & $-343,331.16$ & -2625.64 \\
\hline \multirow[t]{2}{*}{ Buxar } & Discharge & 0.47 & -26.64 & -128.10 & -81.85 & 1.64 \\
\hline & Sediment load & 142.72 & 225.34 & $-26,098.53$ & $-44,982.06$ & 225.00 \\
\hline \multirow[t]{2}{*}{ Gandhighat } & Discharge & 10.84 & -21.05 & -26.46 & 6.85 & 15.75 \\
\hline & Sediment load & 26.30 & 3005.07 & $21,394.68$ & $-65,358.47$ & $25,938.80$ \\
\hline \multirow[t]{2}{*}{ Hathidah } & Discharge & -1.15 & -46.99 & -96.86 & -53.08 & 14.20 \\
\hline & Sediment load & 935.36 & 1360.40 & $-11,127.80$ & $-52,566.35$ & $-11,690.7$ \\
\hline \multirow[t]{2}{*}{ Azamabad } & Discharge & -5.70 & 39.98 & -24.55 & 14.43 & 23.56 \\
\hline & Sediment load & -1858.88 & $-39,710.80$ & $-42,300.40$ & $-74,546.05$ & $-18,890.1$ \\
\hline \multirow[t]{2}{*}{ Farakka } & Discharge & -18.13 & -103.52 & -382.90 & -381.81 & -62.05 \\
\hline & Sediment load & -44.11 & $-27,956.85$ & $-120,687.46$ & $-170,903.79$ & $-59,152.9$ \\
\hline
\end{tabular}

to be $6.2 \%$ which might be attributed to the presence of Farakka Barrage while $3.7 \%$ decrease in sediment load is observed at Ankinghat and Shahzadpur. Table 7 presents the decadal water yield of monsoon season at various gauging sites of Ganga River. It may be observed that as compared to $1970-1980$, the water yield has declined remarkably (13 to $58 \%$ ) in 2000-2010 at most of the gauging sites except Gandhighat.

\section{Spatial pattern of trends}

Trend analysis of sediment yield and water yield of Ganga River reveals a spatial pattern. Analyzing the results reported in Tables 4, 5, and 6 along with Fig. 3, it may be observed that the decline in sediment and water yield was sharper in
Western Ganga Plain as compared to Eastern Ganga Plain. Jain (2008) reported a significant decline in discharge from Gangotri Glacier and concluded that the impact of Gangotri Glacier is effective until Shahzadpur. Decline in discharge of Gangotri Glacier may be considered as the major reason for more pronounced trends in Western Ganga Plain while the decline in discharge of Yamuna River was the major reason for the negative trends observed downstream of Shahzadpur. Spatial trend of annual maximum discharge also reveals a declining trend from Garhmukteshwar to Farakka except Gandhighat. Time series of annual maximum discharge at Gandhighat also revealed an upward trend making it more susceptive to floods. The detailed discussion on monthly trend for each gauging site is provided in the subsequent section. Few graphs obtained from innovative trend analysis have also 
Table 6 Results of ITA of monthly discharge and sediment data

\begin{tabular}{|c|c|c|c|c|c|c|}
\hline Gauging sites & Quantity & June & July & August & September & October \\
\hline \multirow[t]{2}{*}{ Garhmukteshwar } & Discharge & -3.46 & -20.03 & -30.39 & -0.20 & 1.71 \\
\hline & Sediment load & -177.95 & -886.63 & -1334.25 & -33.18 & 22.19 \\
\hline \multirow[t]{2}{*}{ Kachlabridge } & Discharge & 3.08 & -5.93 & -6.54 & -7.31 & 1.24 \\
\hline & Sediment load & 88.81 & 554.06 & 1122.7 & 1086.3 & 296.63 \\
\hline \multirow[t]{2}{*}{ Fatehgarh } & Discharge & 0.04 & -6.78 & -12.16 & 10.70 & 4.33 \\
\hline & Sediment load & -2.14 & -316.15 & -1126.1 & -454.77 & 42.11 \\
\hline \multirow[t]{2}{*}{ Ankinghat } & Discharge & -3.04 & -3.46 & -36.79 & -7.31 & 1.32 \\
\hline & Sediment load & -167.90 & -955.25 & -1126.12 & -1744.17 & -11.75 \\
\hline \multirow[t]{2}{*}{ Kanpur } & Discharge & -3.52 & -20.30 & -27.87 & -13.33 & -5.35 \\
\hline & Sediment load & -3.08 & -574.06 & -1056.85 & -122.50 & -13.20 \\
\hline \multirow[t]{2}{*}{ Bithaura } & Discharge & 3.63 & 8.26 & -9.02 & 2.11 & 10.97 \\
\hline & Sediment load & 4.57 & -19.99 & -840.32 & -1284.11 & 25.35 \\
\hline \multirow[t]{2}{*}{ Shahzadpur } & Discharge & -2.00 & -29.18 & -85.59 & 58.85 & -13.97 \\
\hline & Sediment load & -6.63 & -693.92 & -2210.89 & -1523.45 & -216.89 \\
\hline \multirow[t]{2}{*}{ Allahabad } & Discharge & -0.34 & -62.15 & -221.23 & -177.17 & -21.15 \\
\hline & Sediment load & -88.97 & $-20,895.12$ & $-78,061.25$ & $-39,611.12$ & -7246.01 \\
\hline \multirow[t]{2}{*}{ Mirzapur } & Discharge & 12.89 & 67.84 & -293.96 & -292.54 & -38.53 \\
\hline & Sediment load & 506.75 & $12,289.62$ & $-12,935.51$ & $-22,861.52$ & 6784.81 \\
\hline \multirow[t]{2}{*}{ Varanasi } & Discharge & 1.88 & -49.41 & -147.02 & -153.56 & -34.34 \\
\hline & Sediment load & -7.01 & 2145.63 & $-45,679.81$ & $-35,447.12$ & 341.17 \\
\hline \multirow[t]{2}{*}{ Buxar } & Discharge & -3.79 & 50.50 & -97.16 & -63.18 & 40.46 \\
\hline & Sediment load & 31.90 & 250.32 & $-25,145.20$ & $-15,201.30$ & 120.23 \\
\hline \multirow[t]{2}{*}{ Gandhighat } & Discharge & 20.70 & 18.94 & 109.38 & 99.98 & 3.59 \\
\hline & Sediment load & 38.61 & 36.93 & 62.53 & -1268.93 & 143.07 \\
\hline \multirow[t]{2}{*}{ Hathidah } & Discharge & -10.75 & -26.01 & -89.96 & -30.19 & -6.39 \\
\hline & Sediment load & 34.03 & 43.94 & 266.62 & -935.56 & 15.70 \\
\hline \multirow[t]{2}{*}{ Azamabad } & Discharge & -21.28 & 1.64 & -144.21 & -18.56 & -39.41 \\
\hline & Sediment load & -32.04 & -301.80 & -207.16 & -718.41 & 32.73 \\
\hline \multirow[t]{2}{*}{ Farakka } & Discharge & -6.03 & -64.72 & -359.36 & -353.41 & -105.82 \\
\hline & Sediment load & 180.61 & $-25,793.30$ & $-61,142.32$ & $-128,920$ & $-26,393$ \\
\hline
\end{tabular}

Italicized values represent significant trend at 5\% significance level been presented in subsequent section, because if all the 180 graphs would have been presented, it would have made the article too lengthy.

\section{Garhmukteshwar}

Monthly-trend analysis of discharge presented decreasing trend which was significant during the month of July and August; however, positive trend was observed during October. Sediment load too presented decreasing trend which was significant during the month of June and July. Figure $4 \mathrm{a}$ shows the trend of suspended sediment load for the month of July based on ITA. Monotonic decline in the magnitude of suspended sediment load could be observed from Fig. 4a. Similarly, total water yield and sediment yield during monsoon represent a declining trend at Garhmukteshwar. Decadal monsoon water yield has declined by $36 \%$ from $195,990 \mathrm{Mm}^{3}(1970-1980)$ to $124,806 \mathrm{Mm}^{3}$ (2000-2010). Construction of CCS barrage in Jansath in 1984 could have led to decline in water and sediment discharge.

\section{Kachlabridge}

Monthly discharge generally shows decreasing trend except October. Monthly sediment load generally shows an increasing trend. Total water yield during monsoon season shows a negative trend but the sediment yield represents a positive trend during August, September, and October at Kachlabridge. Decadal monsoon water yield has declined 
Table 7 Total water yield during different durations at various gauging sites of Ganga River

\begin{tabular}{llllll}
\hline Gauging sites & $1960-$ & $1970-$ & $1980-$ & $1990-$ & $2000-$ \\
& 1970 & 1980 & 1990 & 2000 & 2010 \\
\hline Garhmukteshwar & - & 195,990 & 163,097 & 160,528 & 124,806 \\
Kachlabridge & - & 165,975 & 163,285 & 186,272 & 144,269 \\
Fatehgarh & - & - & 137,241 & 187,388 & 124,370 \\
Ankinghat & - & 841,510 & 723,427 & 738,212 & 470,738 \\
Kanpur & 300,513 & 276,810 & 223,267 & 210,212 & 183,038 \\
Bhitaura & & 247,473 & 196,512 & 168,328 & 147,003 \\
Shahzadpur & 336,770 & 312,330 & 226,670 & 151,758 & 143,577 \\
Allahabad & - & $1,261,104$ & 861,914 & 885,541 & 536,108 \\
Mirzapur & - & - & 947,754 & 983,691 & 583,737 \\
Varanasi & 906,247 & $1,033,778$ & 890,296 & 872,658 & 591,507 \\
Buxar & $1,117,954$ & $1,026,088$ & $1,007,621$ & $1,142,893$ & 693,168 \\
Gandhighat & $2,121,211$ & $1,817,068$ & $2,380,834$ & $1,802,690$ & $2,121,211$ \\
Hathidah & $2,347,462$ & $2,631,743$ & $2,328,041$ & $2,552,926$ & $2,148,052$ \\
Azamabad & $2,352,329$ & $3,221,702$ & $2,336,052$ & $2,597,415$ & $2,394,897$ \\
Farakka & - & $3,412,780$ & $3,158,841$ & $3,004,499$ & $2,397,050$ \\
\hline
\end{tabular}

by $13 \%$ from $1970-1980\left(165,975 \mathrm{Mm}^{3}\right)$ to $2000-2010$ $\left(144,269 \mathrm{Mm}^{3}\right)$. Figure $5 \mathrm{a}$ shows the trend of discharge for the month of July based on ITA at Kachlabridge. Based on Fig. 5a, it can be said that the trends in discharge are not monotonic; instead, magnitude of low discharges is declining trend and the magnitude of high discharges is increasing.

\section{Fatehgarh}

Trend analysis of discharge presented decreasing trend which was significant during the month of August. Monthly-trend analysis of sediment load too exhibits a decreasing trend which was significant during the month of July and August; however, positive trend was observed for the month of October. Total water yield and sediment yield during monsoon too represent a negative trend as the river has been divided into a number of channels in the reach.

\section{Ankinghat}

Monthly-trend analysis of discharge presented decreasing trend which is significant during the month of July and August. Sediment load too presented decreasing trend which was significant during the month of July and August. Figure $4 \mathrm{~b}$ shows the trend of suspended sediment load for the month of August. Monotonic decline in the magnitude of suspended sediment load could be observed from Fig. 4b. Total water and sediment yield during monsoon too represent negative trend. Decadal monsoon water yield has declined by $44 \%$ from $841,510 \mathrm{Mm}^{3}$ (1970-1980) to $470,738 \mathrm{Mm}^{3}$ (2000-2010). Ramganga River joins Ganga River between Fatehgarh and Ankinghat. Significant decline in discharge of Ramganga mainly due to decline in rainfall has been reported by Kumar (2017). Decline in discharge of Ramganga has been reflected by significant negative trends in discharge at Fatehgarh and subsequent gauging sites of Ganga.

\section{Kanpur}

Monthly-trend analysis of discharge presented decreasing trend which was significant during the month of July and August. Sediment load too presented remarkable decreasing trend. A sharp decline in sediment load and discharge can be observed due to construction of Lav Khush Barrage in 1995 just upstream of gauging site. Total water yield and sediment yield during monsoon season also represent negative trend at Kanpur. Decadal monsoon water yield has declined by $34 \%$ from $276,810 \mathrm{Mm}^{3}$ (1970$1980)$ to $183,038 \mathrm{Mm}^{3}$ (2000-2010). Figure 5b shows the trend of discharge for the month of August based on ITA at Kanpur. Based on Fig. 5b, it was observed that the magnitude of low discharges is predominantly declining while the magnitude of high discharges has remained almost unchanged.

\section{Bithaura}

Monthly-trend analysis of discharge presented decreasing trend except for October and early June. Total water yield 
a

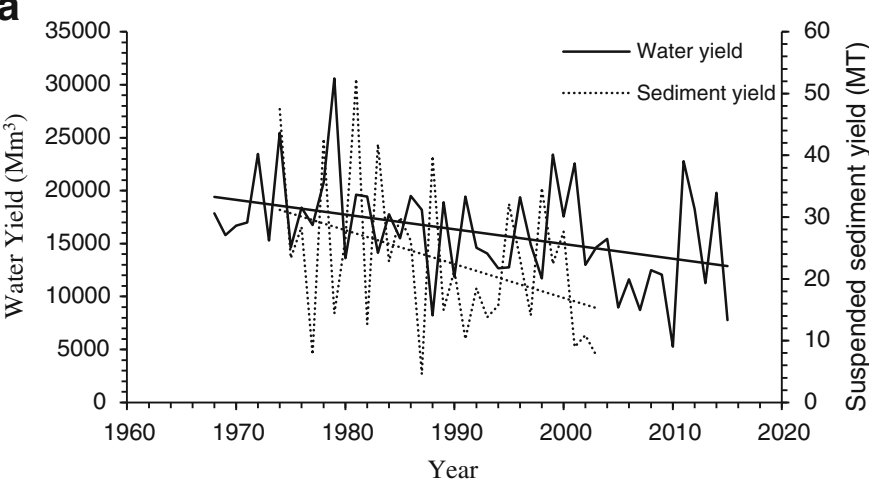

C

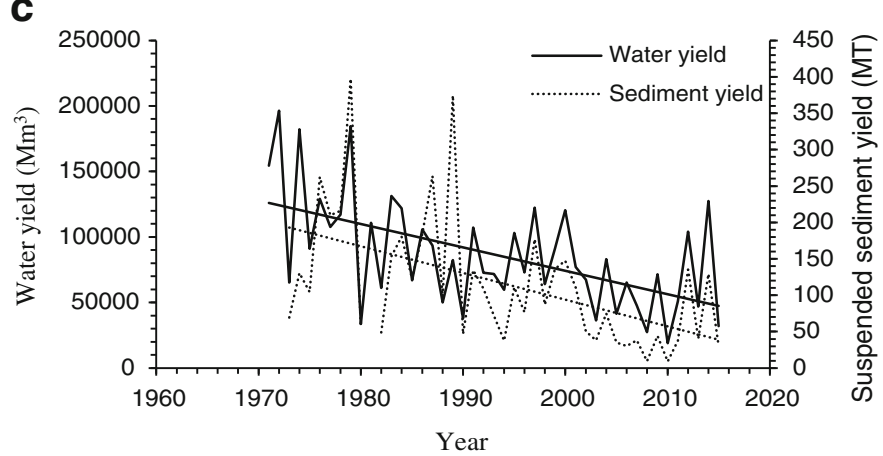

e

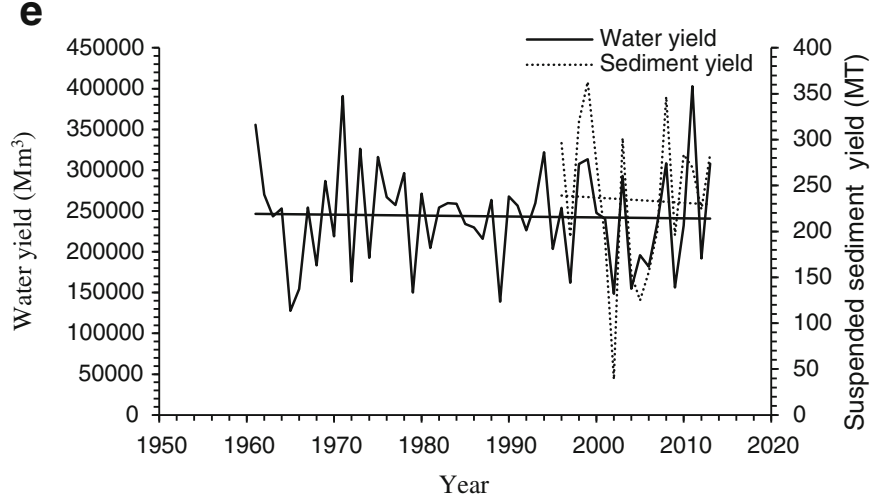

b

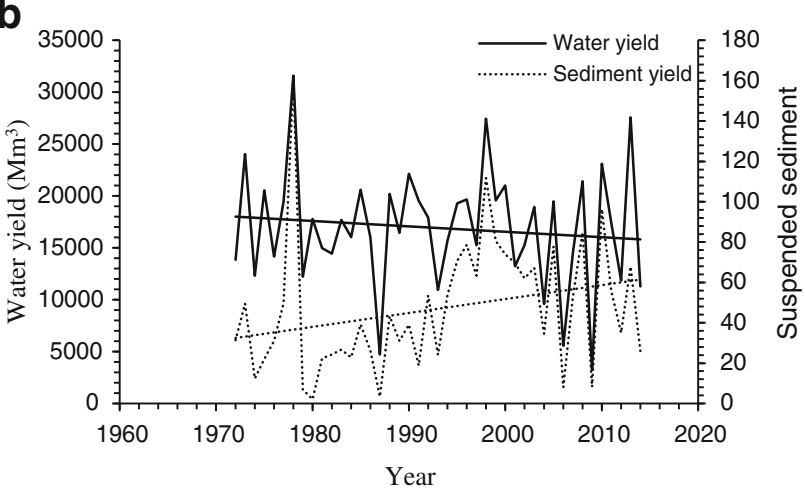

d

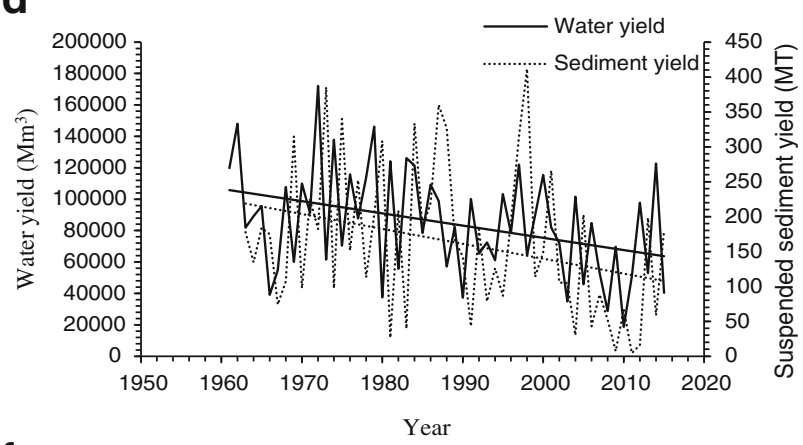

f

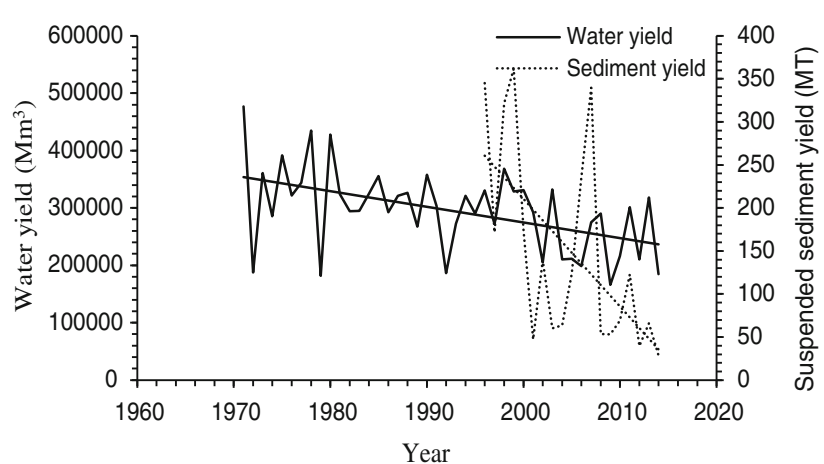

Fig. 3 Trend of water yeild and sediment yeild at a Garhmukteshwar, b Kachlabridge, c Allahabad, d Varanasi, e Hathidah, and f Farakka

and sediment yield during monsoon season also represent negative trend at Bithaura. Decadal monsoon water yield has declined by $40 \%$ from $247,473 \mathrm{Mm}^{3}(1970-1980)$ to 147,003 $\mathrm{Mm}^{3}$ (2000-2010).

\section{Shahzadpur}

Monthly-trend analysis of discharge presented significant decreasing trend. Monthly sediment load presented decreasing trend except for June. Total water yield and sediment yield during monsoon season also represent negative trend at Shahzadpur. Figure $4 \mathrm{c}$ shows the trend of suspended sediment load for the month of September based on ITA. Monotonic decline in the magnitude of suspended sediment load could be observed from Fig. 4c. Decadal monsoon water yield has declined by $54 \%$ from $312,330 \mathrm{Mm}^{3}$ (1970-1980) to $143,577 \mathrm{Mm}^{3}$ (2000-2010).

\section{Allahabad}

Remarkable decrease in discharge and sediment load has been observed from monthly data at Allahabad. Total water yield and sediment yield during monsoon season also represent negative trend at Allahabad. Decadal monsoon water yield has declined by $58 \%$ from $1,261,104 \mathrm{Mm}^{3}(1970-1980)$ to $536,108 \mathrm{Mm}^{3}$ (2000-2010).

\section{Mirzapur}

Decreasing trend was observed in discharge from monthly time series except for the month of June. However, sediment 
a

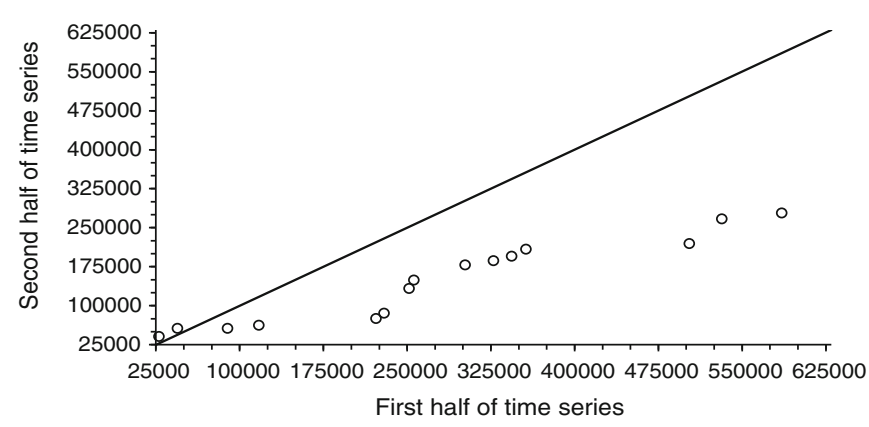

b

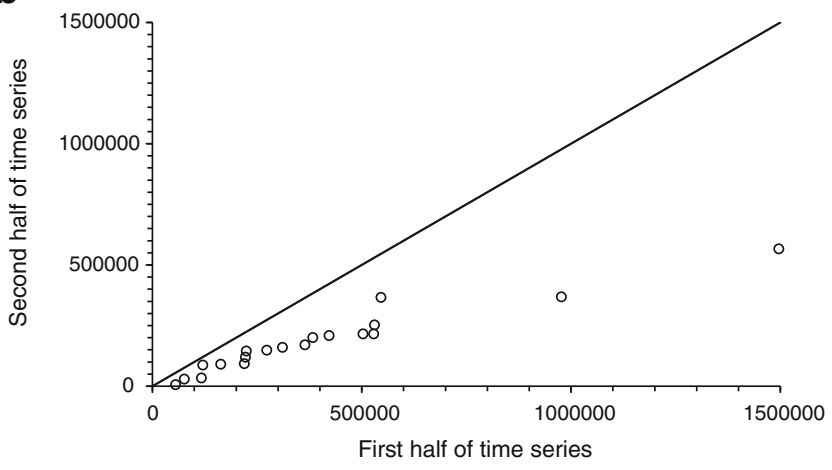

C

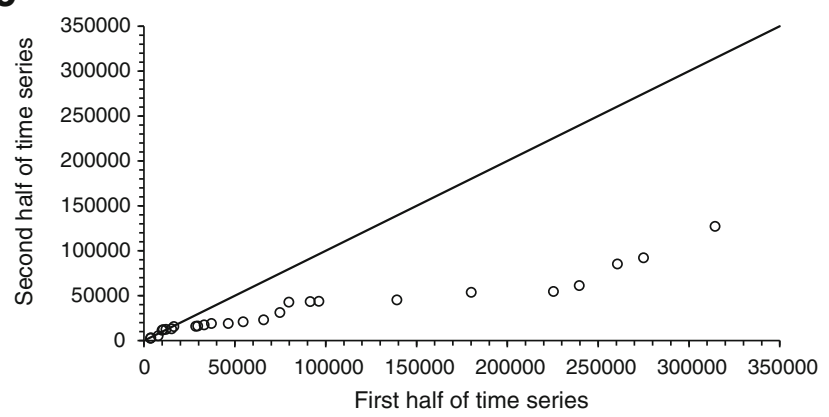

d

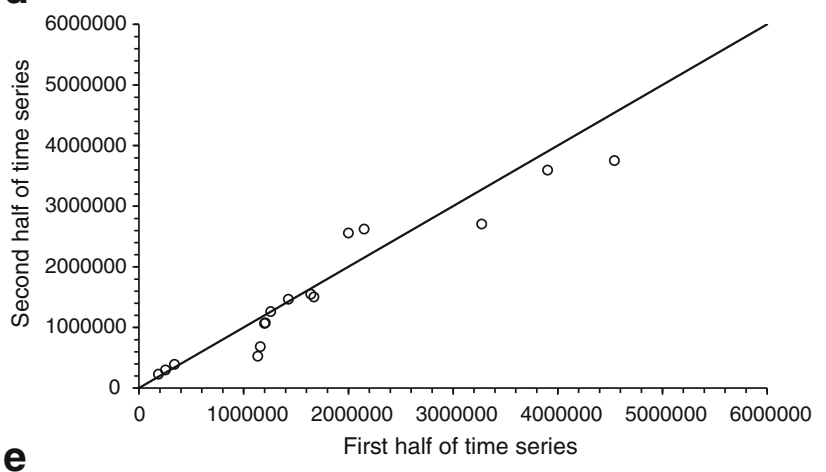

e

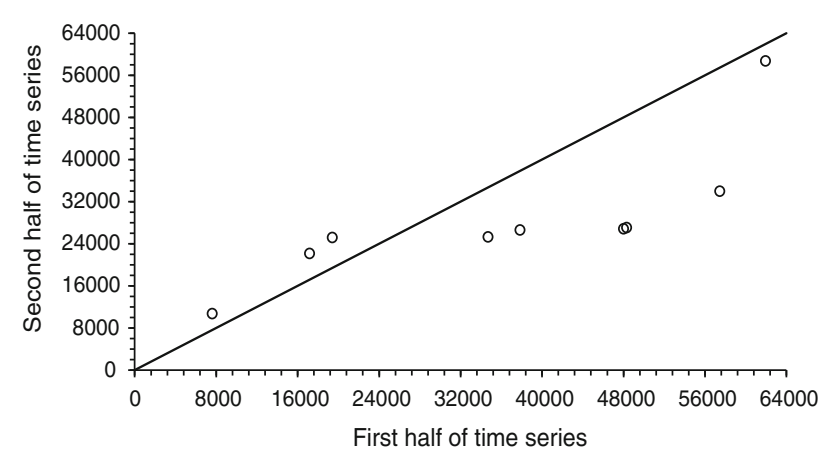

Fig. 4 Trend of suspended sediment load based on ITA at a Garhmukteshwar, b Ankinghat, c Shahzadpur, d Mirapur, and e Hathidah

load presented positive trend during June, July, and October and negative trend during August and September as observed from monthly data. Total water yield and sediment yield during monsoon season also represent negative trend at Mirzapur. Figure $4 \mathrm{~d}$ shows the trend of suspended sediment load while Fig. $5 \mathrm{c}$ shows the trend of discharge for the month of August based on ITA. Figure $5 \mathrm{c}$ shows monotonic decrease in the magnitude of discharge while suspended sediment load was trendless. Decline in rainfall in Tons River basin as reported by Bisht et al. (2018) and Yamuna River could have led to decline in water yield.

\section{Varanasi}

Monthly-trend analysis of discharge presented remarkable decreasing trend; however, positive trend was observed during June. Monthly sediment loads too presented downward trend except for June. Decadal monsoon water yield has declined by
$42 \%$ from $1,033,778 \mathrm{Mm}^{3}$ (1970-1980) to $591,507 \mathrm{Mm}^{3}$ (2000-2010). Bhatla and Tripathi (2014) reported a significant decline in rainfall over Varanasi which may be the reason behind the decline in discharge at Varanasi.

\section{Buxar}

Decreasing trend in discharge was observed during July, August, and September while increasing trend was observed during June and September. Monthly sediment loads too presented downward trend except for June. Total water yield and sediment yield during monsoon season also represent negative trend at Buxar. Decadal monsoon water yield has declined by $32 \%$ from $1,026,088 \mathrm{Mm}^{3}$ (1970-1980) to $693,168 \mathrm{Mm}^{3}$ (2000-2010). Warwade et al. (2018) reported a decline in monsoon rainfall of over $18 \%$ at Buxar which could have led to decline in water yield and other hydrologic parameters. 
a

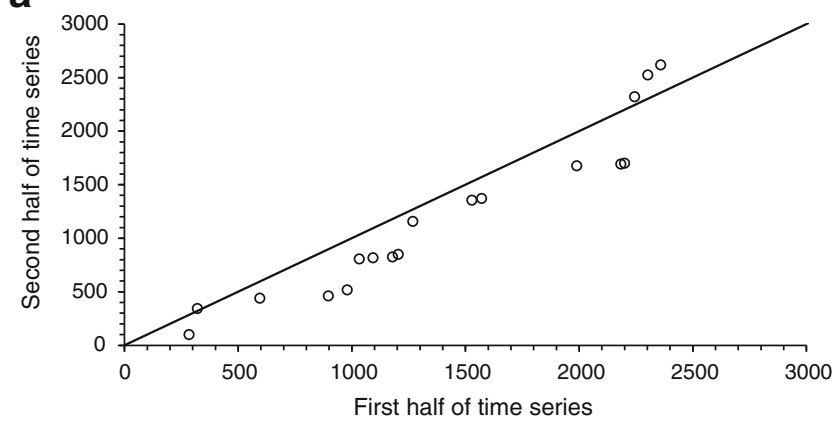

b

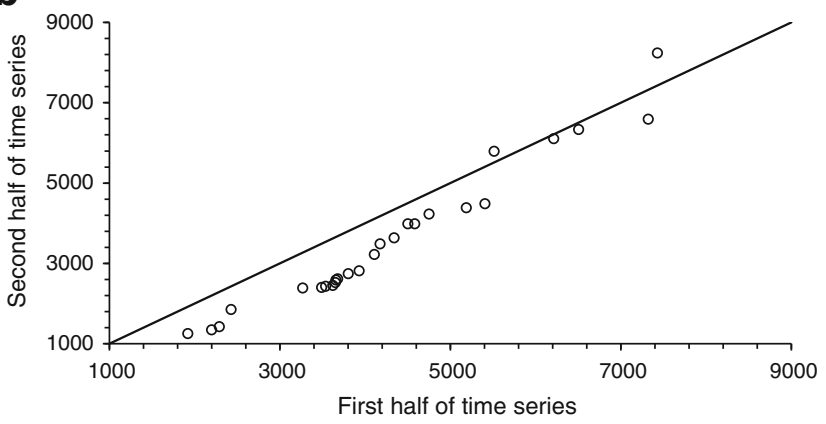

c

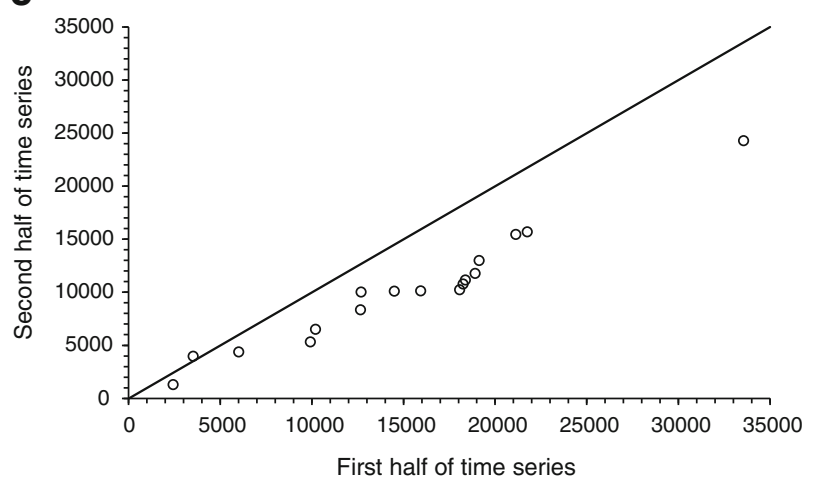

d

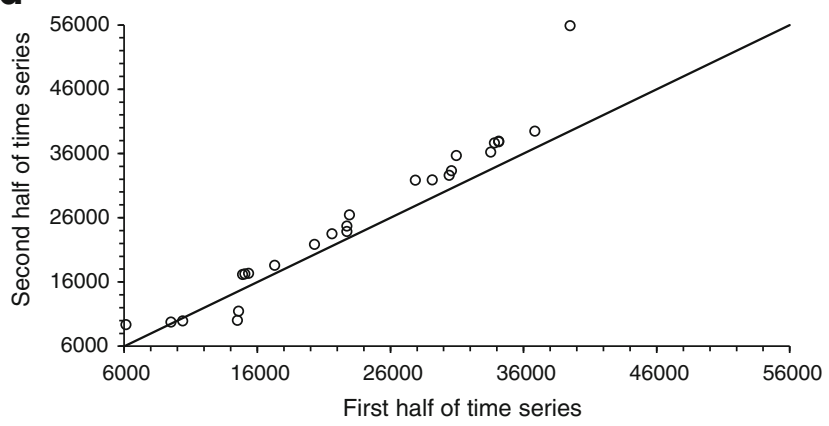

e

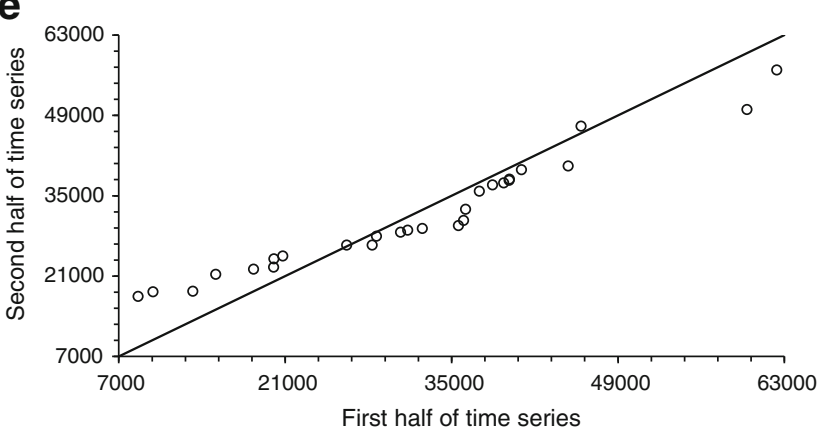

Fig. 5 Trend of discharge based on ITA at a Kaclabridge, b Kanpur, c Mirzapur, d Gandhighat, and e Azamabad

\section{Gandhighat}

Monthly time series shows positive trend during the month of June and October while trends in July, August, and September show mild decreasing trend. Monthly trend of sediment loads represents increasing trend. Total water yield represents an insignificant positive trend while sediment yield represents negative trend at Gandhighat. Rise in annual maximum discharge makes Gandhighat more vulnerable to floods which demands better flood protection and management works to be ensured at Gandhighat. Decadal monsoon water yield has increased by $16 \%$ from $1,817,068 \mathrm{Mm}^{3}$ (1970-1980) to 2,121,211 $\mathrm{Mm}^{3}$ (2000-2010). Between Buxar and Gandhighat, Ghaghara, Sone, and Gandak join the Ganga River. However, trend analysis of available data of these tributaries represents declining trend in general. Figure $5 \mathrm{~d}$ shows the trend of discharge for the month of September based on ITA at Gandhighat. Figure 5d shows monotonic increase in the magnitude of discharge.

Singh et al. (2007) reported that the number of rainy days is decreasing in Ganga River basin. Increase in water yield accompanied by reduction in rainy days is reflected in the form of increase in annual maximum discharge at Gandhighat. Increase in annual maximum discharge at Gandhighat makes this region more susceptive to floods.

\section{Hathidah}

Monthly-trend analysis of average discharge too presented decreasing trend except for the month of October. Sediment load also presented decreasing trend except for the month of June and October. Figure $4 \mathrm{e}$ shows the trend of suspended 
sediment load for the month of September based on ITA. Figure $4 \mathrm{e}$ clearly shows non-monotonic trend in suspended load. Magnitude of low sediment load is increasing; on the other hand, moderate- and high-magnitude sediment loads are declining, resulting in overall declining trend in suspended sediment load. Total water yield and sediment yield during monsoon also represent negative trend at Hathidah. Decadal monsoon water yield has declined by $18 \%$ from 2,631,743 $\mathrm{Mm}^{3}$ (1970-1980) to $2148,052 \mathrm{Mm}^{3}$ (2000-2010).

\section{Azamabad}

Discharge data generally represent upward trend except for the month of June and August. Monthly sediment loads represent a predominant negative trend. Total water yield and sediment yield during monsoon season also represent negative trend at Azamabad. Decadal monsoon water yield has declined by $25 \%$ from $3,221,700 \mathrm{Mm}^{3}$ (1970-1980) to 2,394,900 $\mathrm{Mm}^{3}$ (2000-2010).

Figure 5e shows the trend of discharge for the month of September based on ITA at Azamabad. Figure 5e shows a non-monotonic trend in discharge. At Azamabad, the magnitude of low discharges is increasing; on the other hand, magnitude of high discharges is increasing while the magnitude of moderate discharges has remained unaltered over the period of time.

\section{Farakka}

The discharge and sediment load data represent predominantly negative trend at Farakka. Total water yield and sediment yield during monsoon season also represent negative trend at Farakka. Decadal monsoon water yield has declined by $30 \%$ from $3,412,780 \mathrm{Mm}^{3}$ (1970-1980) to $2,397,050 \mathrm{Mm}^{3}$ (20002010).

Above results indicate that discharge and sediment load are generally decreasing along the Ganga during monsoon season. In Western Ganga Plain, it has been observed that discharge shows upward trend during the month of October. At Farakka, the discharge and sediment load represent the downward trend which may be associated with construction of Farakka Barrage.

In most of the cases, the alteration in sediment yield of rivers has been associated with trapping of sediment load by construction of dams and effective soil erosion control measures (Zhang et al. 2007; Xu and Milliman 2009; Yang et al. 2015; Guo et al. 2018). On the other hand, fluctuation in water yield has been correlated with the alteration of rainfall in river basins (Pham et al. 2019; Tandon et al. 2006; Guo et al. 2018). However, in large river basin, interpretation of hydrologic changes is very difficult because of variety of land surface condition, spatial climatic variation, and human activities (Ives and Messerli 1990; Hofer 1993; Lu et al. 2003).
Supply of sediment load and discharge is triggered by monsoon rains in Ganga River (Zakwan et al. 2018). Monsoon season in the Ganga River basin starts in the month of June, intensifies during July and August, and starts to retreat from mid-September (Zakwan and Ara 2019). Significant negative trends were observed in sediment load and discharge during July and August at most of the gauging sites, thereby deteriorating overall sediment and discharge yield of Ganga River in monsoon. Although sediment and discharge yield of Ganga River depends on large number of factors, yet, decline in monsoon rainfall in Ganga River basin as reported by Bera (2017) may be considered as the major reason. Significant decline in annual rainfall in this region was also reported by Sharma and Ojha (2018) and Sharma et al. (2019). Sharma and Ojha (2018) also detected that the change point in rainfall pattern was 1992 or earlier. Moreover, Moors et al. (2011) and Shrestha et al. (2017) reported an increasing trend in temperature in the Ganga River basin leading to enhanced evaporation rates and decline in discharge.

Ganga basin has also observed significant surge in population and industrial activities, resulting in decline in forest cover and increment in built-up area, thereby contributing to alteration in hydrological response of Ganga basin (Shukla et al. 2017). Surge in industrial activities along with rising population has led to construction of dams, barrages, and canal across the Ganga and its tributaries to encounter the increased requirements of energy and food, leading to alteration in flow. Ganga basin is also experiencing a significant increase in construction activities which has led to increased rate of mining from Ganga River and its tributaries which is adversely affecting the morphology of the river (Barman et al. 2019; Park et al. 2020). The flow of the river is also influenced by rituals. Comparative pictures of Ganga, before and after the 21-day COVID-19 lockdown, are also evidence of human intervention with the natural system of Ganga River.

Hence, it may be concluded that both climate change and human intervention have contributed to changes in flow pattern and sediment flux in the Ganga River basin. Generally, it is assumed that sediment load is directly proportional to discharge through power law; therefore, decline in sediment flux with decline in discharge is obvious.

Ganga River basin being the largest and most populous basin of India is the source of livelihood of millions of people. Alteration in flow pattern of Ganga River would have farreaching socio-economic consequences for the entire region. Many cities lying on the bank of Ganga River have already started experiencing shortage of water during the summer season. Decline in groundwater table has also been common in many parts of the river basin over the past few years. With increased effluents from industries, water quality in the region has declined remarkably, thereby affecting aquatic life and self-cleansing capability of the river. Sarkar et al. (2012) have reported that many species of fishes, earlier found in Ganga 
River, have become extinct. Sarkar et al. (2012) also reported an overall decline in the fishes in Ganga River which has led to a huge loss to fisheries sector. Decline in discharge as observed in the present study would also lead to decline in hydropower production of hydropower stations of the country.

Despite numerous measures to rejuvenate and manage Ganga River, the health of the Ganga is declining at an alarming rate basically because restoration of Ganga River requires an integrated approach which requires combined efforts of hydrologists, geomorphologists, ecologists, environmental experts, social workers, politicians, and most essentially local people. Ganga River is marked by diverse geographical, climatic, and morphological setting (Sinha et al. 2017). In this regard, Ganga River management strategy would essentially involve diverse rejuvenation measures for different reaches. As an example, Roy and Sinha (2014) and Zakwan et al. (2018) reported that bankfull discharge is a rare event in Western Ganga Plain and Ganga River is subjected to deep incision in this region, but around Patna, bankfull discharge is a frequent event and this section of Ganga requires special attention from the prospect of flood protection works.

\section{Conclusion}

Trend analysis was performed using the Mann-Kendall, Sen's slope, and innovative trend tests of different hydrologic parameters at various gauging sites located on the Ganga River. It was observed that almost all the parameters, like annual maximum and annual minimum discharges, sediment and water yield crossing gauging sites during monsoon, showed a negative trend at 5\% significance level indicating a fall in all the parameters with time. The presence of significant negative trend at gauging sites downstream of Yamuna from Allahabad to Buxar calls for detailed study of the behavior of the major tributary Yamuna. A significant negative trend in discharge and sediment yield was observed at Farakka which may be attributed to the presence of barrage at the location. Contrary to the general negative trend, Gandhighat exhibit a positive trend behavior for annual maximum and annual minimum discharge, average discharge, and sediment load. Innovative trend analysis could reveal trends of different magnitudes of events; in this way, its application is certainly advantageous over the traditional Mann-Kendall and Sen's slope tests.

Hydrology of large river basin such as Ganga is influenced by various land surface condition, spatial climatic variation, and human activities and as such, it is very difficult to ascertain a particular reason for alteration in follow pattern. However, as evident from the present and earlier studies, both climatic factors (changes in the rainfall and temperature) and human intervention (changes in land use pattern, demographic changes, industrial activities, construction of hydraulic structures) have influenced the flow pattern of Ganga.
Ganga River is marked by diverse geographical, climatic, and morphological setting. In this regard, Ganga River management strategy would essentially require integrated approach with emphasis on diverse rejuvenation measures for different reaches of Ganga River.

Acknowledgments The authors would like to acknowledge the Central Water Commission (CWC) for providing data for the project.

Funding The authors would like to thank the Ministry of Water Resources (MoWR), India, for funding this project.

Data availability Some or all data, models, or code used during the study were provided by a third party. Direct requests for these materials may be made to the provider as indicated in the Acknowledgments.

\section{Compliance with ethical standards}

Conflict of interest The authors declare that they have no conflict of interest.

\section{References}

Abbas N, Subramanian V (1984) Erosion and sediment transport in the Ganga river basin, India. J Hydrology 69:173-182

Arora M, Kumar R, Kumar N, Malhotra J (2014) Assessment of suspended sediment concentration and load from a large Himalayan glacier. Hydrol Res 45(2):292-306

Barman, S. D., Islam, A., Das, B. C., Mandal, S., \& Pal, S. C. (2019). Imprints of Neo-tectonism in the evolutionary record along the course of Khari river in Damodar fan delta of lower Ganga basin. In Quaternary geomorphology in India (pp. 105-126). Springer, Cham

Bhatla R, Tripathi A (2014) The study of rainfall and temperature variability over Varanasi. Int J Earth Atmos Sci 1(2):90-94

Benzater B, Elouissi A, Benaricha B, Habi M (2019) Spatio-temporal trends in daily maximum rainfall in northwestern Algeria (Macta watershed case, Algeria). Arab J Geosci 12(11):370

Bisht DS, Chatterjee C, Raghuwanshi NS, Sridhar V (2018) Spatiotemporal trends of rainfall across Indian river basins. Theor Appl Climatol 132(1-2):419-436

Chakrapani GJ, Saini RK (2009) Temporal and spatial variations in water discharge and sediment load in the Alaknanda and Bhagirathi Rivers in Himalaya, India. J Asian Earth Sci 35(6):545-553

Ebadati N, Hooshmandzadeh M, Behzad N (2014) A comparison of the correlation Matrix and Man-Kendal correlation statistical methods for analyzing the qualitative parameters of Dez River water. J Magnt Res Rep 2:986-1001

Elouissi A, Habi M, Benaricha B, Boualem SA (2017) Climate change impact on rainfall spatio-temporal variability (Macta watershed case, Algeria). Arab J Geosci 10(22):496

Elouissi A, Șen Z, Habi M (2016) Algerian rainfall innovative trend analysis and its implications to Macta watershed. Arab J Geosci 9(4):303

Guo LP, Yu Q, Gao P, Nie XF, Liao KT, Chen XL, Mu XM (2018) Trend and change-point analysis of streamflow and sediment discharge of the Gongshui River in China during the last 60 years. Water 10(9): 1273

Hofer T (1993) Himalayan deforestation, changing river discharge, and increasing floods: myth or reality? Mt Res Dev 13:213-233 
Ives, J. D., \& Messerli, B. (1990). Progress in theoretical and applied mountain research, 1973-1989, and major future needs. Mountain Research and Development, 101-127

Jain SK (2008) Impact of retreat of Gangotri glacier on the flow of Ganga River. Curr Sci 95(8):1012-1014

Kendall MG (1975) Rank correlation methods. Griffin, London, UK

Khazaei S, Barati R, Ghandehary A, Sadeghifard MR (2019) Rainfall trend analysis using innovative-Șen method and comparison with traditional methods (case study: Khorasan Razavi province). J Water Sustain Dev 6(1):41-50

Khattab MF, Merkel BJ (2014) Application of Landsat 5 and Landsat 7 images data for water quality mapping in Mosul Dam Lake, Northern Iraq. Arab J Geosci 7(9):3557-3573

Kumar, R. (2017). Flow characterization of Ramganga River catchment through hydrologic models (Doctoral dissertation, Department of Farm Engineering, Institute of agricultural sciences, BHU, Varanasi-5)

Lu XX, Ashmore P, Wang JF (2003) Seasonal water discharge and sediment load changes in the Upper Yangtze, China. Mt Res Dev 23(1): $56-64$

Mann HB (1945) Nonparametric test against trend. Econometrica. 13: 245-259

Milliman JD, Farnsworth KL, Jones PD, Xu KH, Smith LC (2008) Climatic and anthropogenic factors affecting river discharge to the global ocean, 1951-2000. Glob Planet Chang 62(3-4):187-194

Millimen JD, Meade RH (1983) Worldwide delivery of river sediments to the oceans. J Geol 91(1):1-21

Misra AK (2010) River about to die: Yamuna. Water Resour Prot 2(5): $489-500$

Moors EJ, Groot A, Biemans H, Scheltinga CTV, Siderius C, Stoffel M, Huggel C, Wiltshire A, Mathison C, Ridley J, Jacob D, Kumar P, Bhadwal S, Gosain A, Collins DN (2011) Adaptation to changing water resources in the Ganges basin, northern India. Environ Sci Pol 14:758-769

Muzzammil M, Alam J, Zakwan M (2018) A spreadsheet approach for prediction of rating curve parameters, In Hydrologic modeling (pp. 525-533). Springer, Singapore

Pandey M, Zakwan M, Sharma PK, Ahmad Z (2018) Multiple linear regression and genetic algorithm approaches to predict temporal scour depth near circular pier in non-cohesive sediment. ISH J Hydraul Eng:1-8

Park E, Loc HH, Dung TD, Yang X, Alcantara E, Merino E, Son VH (2020) Dramatic decrease of flood frequency in the Mekong Delta due to river-bed mining and dyke construction. Sci Total Environ 723:138066

Pham QB, Yang TC, Kuo CM, Tseng HW, Yu PS (2019) Combing random forest and least square support vector regression for improving extreme rainfall downscaling. Water 11(3):451. https://doi.org/ $10.3390 /$ w1 1030451

Roy NG, Sinha R (2007) Understanding confluence dynamics in the alluvial Ganga-Ramganga valley, India: an integrated approach using geomorphology and hydrology. Geomorphology. 92:182-197

Roy NG, Sinha R (2014) Effective discharge for suspended sediment transport of the Ganga River and its geomorphic implication. Geomorphology. 227:18-30

Sarkar UK, Pathak AK, Sinha RK, Sivakumar K, Pandian AK, Pandey A, Dubey VK, Lakra WS (2012) Freshwater fish biodiversity in the River Ganga (India): changing pattern, threats and conservation perspectives. Rev Fish Biol Fish 22(1):251-272

Sen PK (1968) Estimates of regression coefficient based on Kendall's tau. J Am Stat Assoc 63:1379-1389
Şen Z (2012) Innovative trend analysis methodology. J Hydrol Eng 17(9): $1042-1046$

Şen Z (2017) Innovative trend significance test and applications. Theor Appl Climatol 127(3-4):939-947

Singh, M., Singh, I. B., and Müller, G. (2007). Sediment characteristics and transportation dynamics of the Ganga River. Geomorphology, 86(1-2), 144-175.

Sharma, C., \& Ojha, C. S. P. (2018). Climate change detection in Upper Ganga river basin. In Proceedings of the International Conference on Sustainable Technologies for Intelligent Water Management, Roorkee, India (pp. 16-19).

Sharma C, Ojha CSP, Shukla AK, Pham QB, Linh NTT, Fai CM, Ho HL, Dung TD (2019) Modified approach to reduce GCM bias in downscaled precipitation: a study in Ganga River basin. Water 11:2097

Shrestha AB, Bajracharya SR, Sharma AR, Duo C, Kulkarni A (2017) Observed trends and changes in daily temperature and precipitation extremes over the Koshi river basin 1975-2010. Int J Climatol 37: 1066-1083

Shukla AK, Pathak S, Pal L, Ojha CSP, Mijic A, Garg RD (2017) Spatiotemporal assessment of annual water balance models for upper Ganga Basin. Hydrol Earth Syst Sci Discuss 536:1-29

Siakeu J, Oguchi T, Aoki T, Esaki Y, Jarvie HP (2004) Change in riverine suspended sediment concentration in central Japan in response to late 20th century human activities. Catena 55(2):231-254

Sinha R, Friend PR (1994) River systems and their sediment flux, IndoGangetic Plains, Northern Bihar, India. Sedimentology 41:825-845

Sinha R, Mohanta H, Jain V, Tandon SK (2017) Geomorphic diversity as a river management tool and its application to the Ganga River, India. River Res Appl 33(7):1156-1176

Subramanian, V., (1996). The sediment load of Indian rivers - an update. Erosion and Sediment Yield: Global and Regional Perspective: Proceedings of the Exeter Symposium. IASH Pub. No. 236.

Tandon SK, Gibling MR, Sinha R, Singh V, Ghazanfari P, Dasgupta A, Jain M, Jain V (2006) Alluvial valleys of the Gangetic Plains, India: causes and timing of incision. SEPM Spec Publ 85:15-35

Tirkey N, Parhi PK, Lohani AK, \& Chandniha SK (2020) Analysis of precipitation variability over Satluj Basin, Himachal Pradesh, India: 1901-2013. J Water Clim Change.

Walling DE, Fang D (2003) Recent trends in suspended sediment loads of the world's rivers. Glob Planet Chang 39:111-126

Warwade P, Tiwari S, Ranjan S, Chandniha SK, Adamowski J (2018) Spatio-temporal variation of rainfall over Bihar State, India. J Water Land Dev 36(1):183-197

Xu K, Milliman JD (2009) Seasonal variations of sediment discharge from the Yangtze River before and after impoundment of the Three Gorges Dam. Geomorphology 104(3-4):276-283

Yang SL, Xu KH, Milliman JD, Yang HF, Wu CS (2015) Decline of Yangtze River water and sediment discharge: impact from natural and anthropogenic changes. Sci Rep 5:12581

Yue S, Pilon P, Phinney B, Cavadias G (2002) The influence of autocorrelation on the ability to detect trend in hydrological series. Hydrol Process 16:1807-1829

Zhang S, Lu XX, Higgitt DL, Chen CTA, Han H, Sun H (2007) Recent changes of water discharge and sediment load in the Zhujiang (Pearl River) Basin, China. Glob Planet Chang 60:365-380

Zakwan M (2018) Spreadsheet-based modelling of hysteresis-affected curves. Appl Water Sci 8(4):101-105

Zakwan M, Ara Z (2019) Statistical analysis of rainfall in Bihar. Sustain Water Resour Manag 5(4):1781-1789

Zakwan M, Ahmad Z, Sharief SMV (2018) Magnitude-frequency analysis for suspended sediment transport in the Ganga River. J Hydrol Eng 23(7):05018013 